\title{
Study on the Location of mmWave Antenna for the Autonomous Car's Detection and Ranging Sensors
}

\author{
Ali Araghi, Mohsen Khalily, Pei Xiao, Rahim Tafazolli \\ Institute for Communication Systems, Home of 5G Innovation Centre, University of Surrey, Guildford, UK \\ \{a.araghi, m.khalily, p.xiao, r.tafazolli\}@ surrey.ac.uk
}

\begin{abstract}
The effect of vehicle's proximity on the radiation pattern when the RADAR's antenna is mounted on the body of autonomous cars is analysed. Two directional radiation patterns with different specifications are placed at different locations of a realistic car body model. The simulation is performed based on ray-tracing method at $77 \mathrm{GHz}$, the standard frequency for selfdriving applications. It is shown that to obtain a robust RADAR sensor, the antenna radiation pattern is better to have relatively higher gain and lower side-lobe-level (SLL), than narrower halfpower-beamwidth (HPBW) and higher front-to-back (F/B) ratio. Both academia and industry can benefit from this study.
\end{abstract}

Index Terms-Self-driving vehicles, RADAR sensors, scattering, ray tracing, millimetre wave.

\section{INTRODUCTION}

With the emergence of $5 \mathrm{G}$ network and IoT system, selfdriving cars have an obvious chance to become industrialized and hit the market in the near future [1]. Autonomous cars rely on the data gathered from different sensing systems. First of all, video cameras are to detect traffic lights, signs, and to capture the position of other obstacles [2], [3]. Then, light detection and ranging (LIDAR) sensors are applied to analyse and identify lane markings and the edges of the roads [1]. After that, ultrasonic sensors can be used to measure the position of objects very close to the car [4]. Last but not least, radio detection and ranging (RADAR) sensors are used to monitor the position of other vehicle nearby [1], [5]. The accurate and reliable performance of the RADAR system is necessary since the safety of passengers will depend on it directly. In order to achieve a well-performance forward-looking RADAR system, directional-beam antenna is required to spot the target accurately [6], [7]. But where the antenna should be located on the car's body and what features are more important for the directive beam?

Two main allocated bands for automotive RADAR systems are $24 \mathrm{GHz}$ and $77 \mathrm{GHz}$ [1], [5]. It is easier to develop a $24 \mathrm{GHz}$ antenna, but more challenging to integrate such a RADAR system into the vehicle due to its relatively larger size. As a result, the $77 \mathrm{GHz}$ RADAR is preferred. In addition, the $77 \mathrm{GHz}$ band provides a better resolution of objects and increases the detection accuracy. However, in the higher band, the radiation pattern of RADAR's antenna is more likely to be affected by the housing around it, i.e. the car's body itself. Due to the smaller wavelength at $77 \mathrm{GHz}$, the radiating waves will be scattered by any small curvatures on the vehicle's body and modern cars generally possess lots of these curves to meet the market's expectations.

RADAR's antenna location for future self-driving cars has been a controversial topic [6] and to the best of our knowledge there is no comprehensive analysis on it so far. In this paper, a study on the effect of vehicle's body on the radiation pattern of the RADAR's antenna for self-driving applications is conducted. The focus is on the $77 \mathrm{GHz}$ band since as mentioned before, it is more practical and also more sensitive to where exactly the antenna is going to be mounted on the car's body.

\section{Methodology}

The first task is to generate a directional beam as it is required for the RADAR sensors. Therefore, two conventional antennas, capable of providing relatively high-gain beams are simulated using full-wave package ANSYS HFSS. These antennas are an $8 \times 7$ planar array of standing-wave slotted substrate integrated waveguide (SIW) [8] and a pyramidal horn antenna (see Fig. 1 (a) and (b)). In order to conduct a comprehensive study about the vehicle effect on the radiating beam, the antennas are designed in a way to generate two directional radiation patterns with different characteristics. These characteristics are gain, half-power-beam-width (HPBW), side-lobelevel (SLL), and front-to-back (F/B) ratio.

Using two different antennas provides more freedom to achieve different radiation characteristics. For example, if just the slotted SIW array antenna is used, altering the gain could be performed by changing the number of elements. It is also possible to control the SLL by tapering the slots location. However, it would be a challenging task to vary the F/B ratio considerably. On top of that, the aim of this paper is to study the effect of car's body on the radiation performance, not to design antennas with some specific features.

The aforementioned antennas characteristics are as follows;

- $8 \times 7$ Slotted SIW Array: Alumina $99.5 \%$ with thickness of $h=0.254 \mathrm{~mm}$ is applied as the substrate of this antenna because of its extremely low $\tan \delta$ which helps the antenna's aperture be illuminated properly at $77 \mathrm{GHz}$. Antenna's radiation pattern is presented in Fig. 1 (c) and (e). The number of elements are chosen in a way to achieve a directional beam with $16 \mathrm{dBi}$ gain. The $\mathrm{H}$-plane (E-plane) antenna characteristics are $\mathrm{HPBW}=12 \mathrm{deg}$ $(\mathrm{HPBW}=24 \mathrm{deg}), \mathrm{SLL}=-10.5 \mathrm{~dB}(\mathrm{SLL}=-21 \mathrm{~dB})$, and $\mathrm{F} / \mathrm{B}=40.8 \mathrm{~dB}$. 

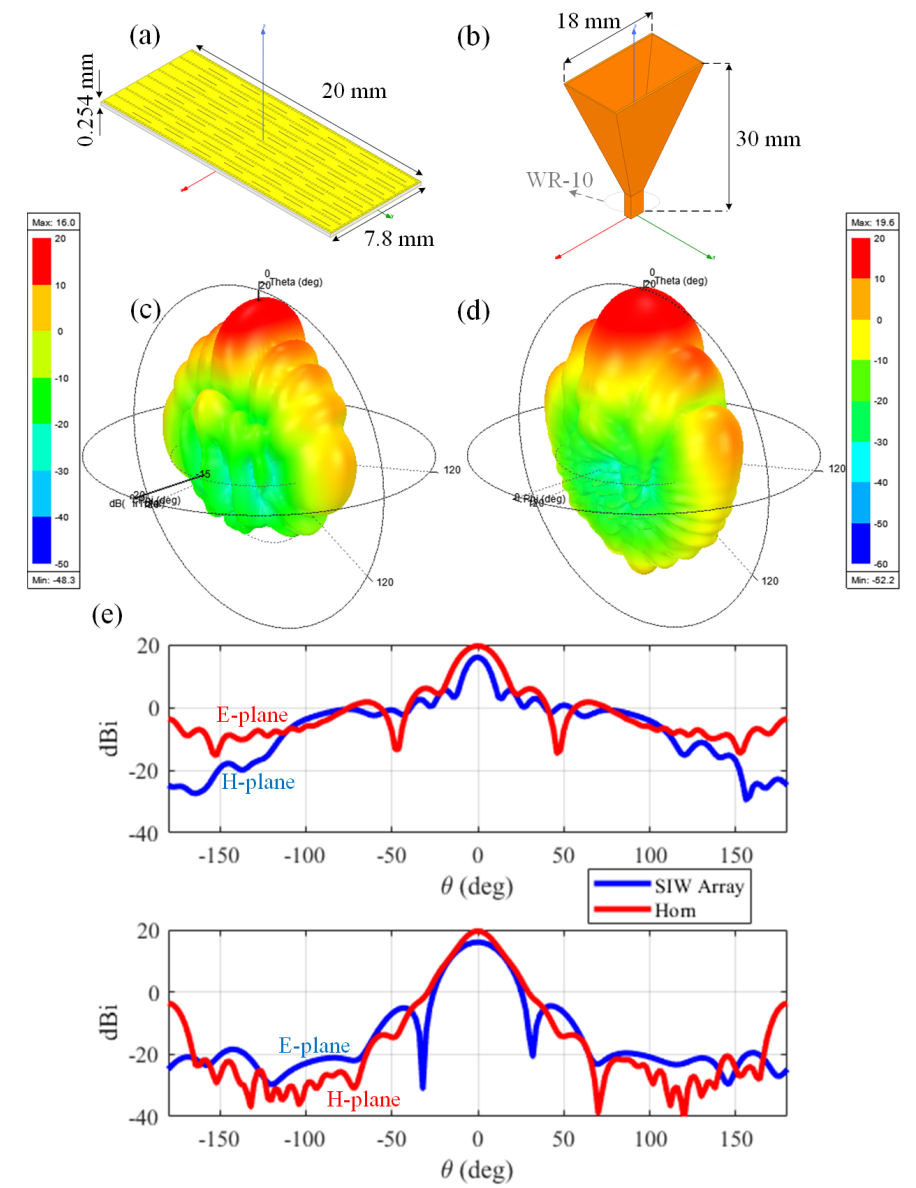

Fig. 1. The simulated antennas and their corresponding radiation patterns. (a) the slotted array on SIW, (b) the pyramidal horn, (c) radiation pattern of the slotted array on SIW, (d) radiation pattern of the pyramidal horn, (e) E-plane and H-plane gains.

- Pyramidal Horn: WR-10 waveguide is used as the base of the pyramidal horn while the radiating aperture's dimensions is managed in a way to achieve 19.6 dBi gain, higher than the previous antenna. Antenna's radiation pattern is presented in Fig. 1 (d) and (e). The E-plane (H-plane) antenna characteristics are $\mathrm{HPBW}=20 \mathrm{deg}$ $(\mathrm{HPBW}=17 \mathrm{deg}), \mathrm{SLL}=-13.6 \mathrm{~dB}(\mathrm{SLL}=-33.5 \mathrm{~dB})$, and $\mathrm{F} / \mathrm{B}=23.1 \mathrm{~dB}$.

Table I provides a comparison between the two generated radiation patterns as the reference of this study. As it is clear from the table, the positive features are divided between the antennas equally, enables us to end up with a conclusion on what feature will have a more prominent influence throughout this study.

Next task is to locate the antenna on the car's body in a way to enable the car sensing its front. Eight places are defined to mount the antenna on the vehicle's body as presented in Fig. 2. The scattering effect of the automobile on the radiation pattern is simulated by the ANSYS Savant. This software is based on the ray-tracing method, therefore it can analyse the radiation performance of electrically large platforms. The first step is
TABLE I

Comparison Between The Two Generated Radiation Patterns

\begin{tabular}{l|l|l} 
& Slotted SIW Array & Pyramidal Horn \\
\hline \hline Higher gain & & $\checkmark$ \\
\hline Lower HPBW & $\checkmark$ & \\
\hline Lower SLL & & $\checkmark$ \\
\hline Lower F/B & $\checkmark$ &
\end{tabular}

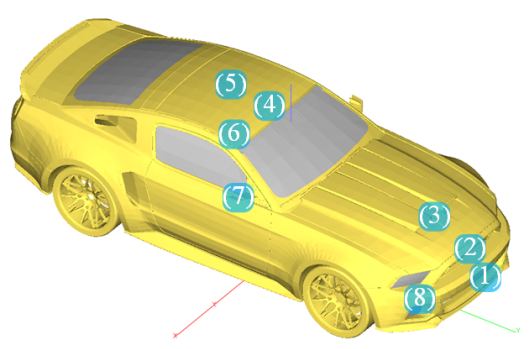

Fig. 2. Different scenarios for the $77 \mathrm{GHz}$ RADAR's antenna placing on the vehicle's body.

to import the simulated radiation pattern from the HFSS to Savant and then to import the realistic model of a car in the ongoing project. Several free CAD models can be found in [9]. A realistic model of the Ford Mustang-GT is chosen since its relatively curved and twisted body provides many sources of scattering, makes it the worst case of study (excluding military vehicles or Formula-1 cars).

The imported car's body is covered by aluminum while the ceramic glass with thickness of $5.76 \mathrm{~mm}$ and $\epsilon_{r}=11$ [10] is assigned for windshields, lateral windows, and headlights. The area inside the cabin is set to be empty. Since the tires are electrically far from the antenna's location in all scenarios (see Fig. 2), their effect on the radiation pattern can be completely ignored in the $77 \mathrm{GHz}$ band. Therefore, to reduce the simulation processing time, no new material is set for the tires.

\section{RESUlTS AND Discussions}

Figure 3 (a) and (b) show the ray shooting and bouncing procedure at rendering frequency of $77 \mathrm{GHz}$ when the slotted SIW array is placed at the locations (2) and (3) of Fig. 2. Comparing Fig. 3 (a) and (b), it can be observed that the number of reflected rays are relatively high in the former case, while for the latter one, a considerable portion of energy is wasted back toward the windshield. Fig. 3 (c) is obtained when the pyramidal horn is located in location (1) of Fig. 2. As it is clear, the energy is radiated to the front of vehicle with less number of bounced rays.

The distorted radiation pattern, when the slotted SIW is located at position (1) (see Fig. 2) in a way that its $\mathrm{H}$ plane is aligned first vertically and then horizontally with respect to the car's coordination are presented in Fig. 4 (a) and (b) respectively. The former figure shows that the sidelobes of the initial radiation pattern (Fig. 1 (c)) are amplified 


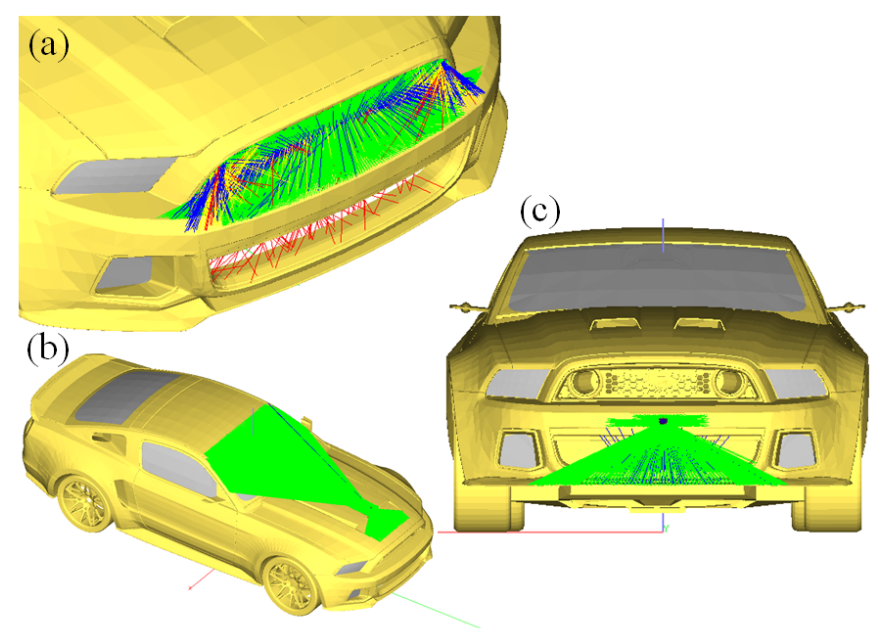

Fig. 3. The ray shooting and bouncing procedure at rendering frequency of $77 \mathrm{GHz}$. (a) and (b): the slotted SIW array is placed at the location (2) and (3) of Fig. 2 respectively, (c) the pyramidal horn in location (1) of Fig. 2.

inconveniently, resulting in a multiple beam pattern. This pattern cannot be used in RADAR systems.

Considering Fig. 4 (b), the constructed radiation pattern is more like a fan-beam, rather that a spot-beam; the main lobe is significantly shrunk and therefore horizontally aligned radiation pattern is not be an option at all. Fig. 4 (c) $\sim$ (i) are representing the distorted radiation patterns when the vertically-aligned initial radiation pattern is located at positions $(2) \sim(8)$ respectively.

With respect to Fig. 4 (c) and Fig. 3 (a), the negative effect of multiple bouncing on the radiation pattern can be observed. The RADAR's antenna must never be located in such a cavityshaped regions on the vehicle's body. Fig. 4 (d) $\sim$ (f) show some other impractical locations of positioning the initial radiation pattern. Fig. 4 (g) (location number 6 in Fig. 2) shows that although the radiation pattern is distorted, the beam is more directional in comparison with the previous locations. Finally, Fig. 4 (h) and (i) (locations 7 and 8) present two cases in which the radiation pattern, more or less remains similar to its initial form of Fig. 1 (c). At these locations the antenna is almost outside the vehicle, i.e. on the side-mirror and bumper's corner.

Next study is to analyse the effect of vehicle proximity on the pyramidal horn's radiation pattern. Regarding the locations 1, 3, 4, 5, 7, and 8 in Fig. 2, the simulated results are presented in Fig. 5 (a) $\sim$ (f) respectively. This figure shows that the constructed radiation pattern is not distorted dramatically in all these locations.

Referring to Table I, Fig. 4, and Fig. 5 it can be concluded that higher gain and lower SLL are more important parameters to be considered rather than HPBW and F/B ratio for this specific application. Mounting an antenna on the vehicle with radiation pattern like Fig. 1 (c) requires extra attentions since just two locations of (7) and (8) will result in an appropriate performance. However, an antenna with radiation
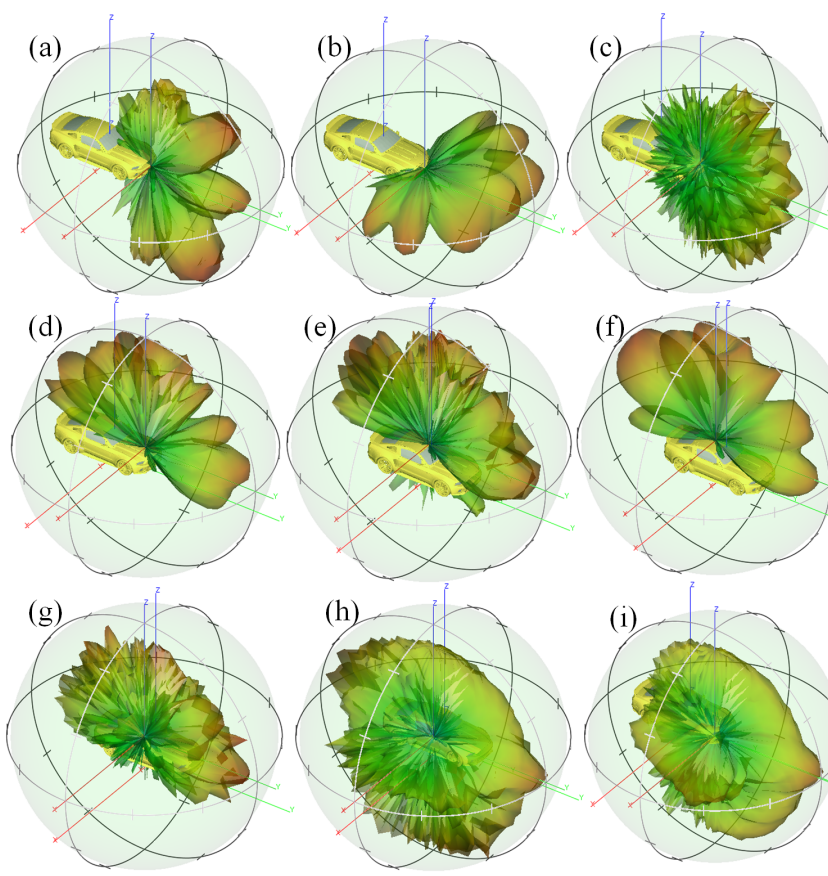

(h)
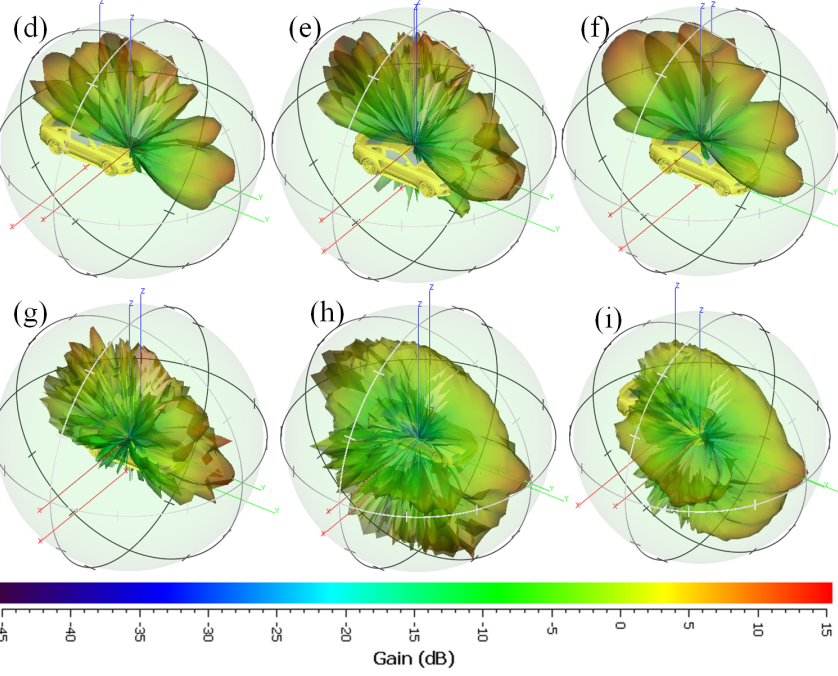

Fig. 4. The scattered radiation pattern. (a) and (b): slotted SIW array is located at position (1) of Fig. 2 vertically and horizontally with respect to the H-plane. (c) (i): the antenna (vertically aligned) is located at positions (2) $\sim(8)$ respectively.
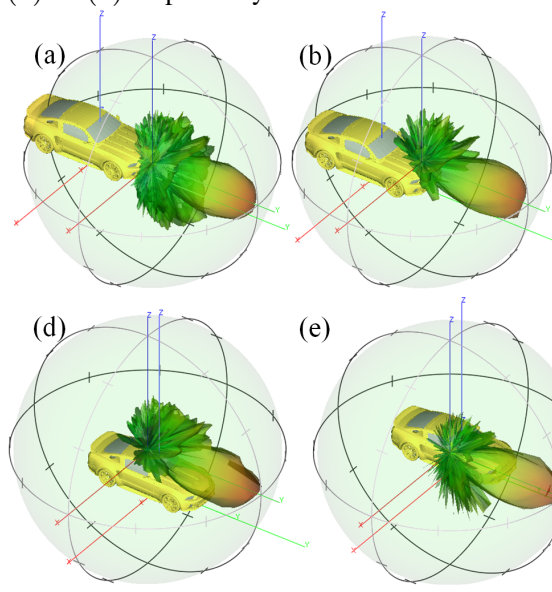

(e)

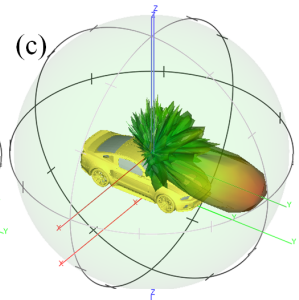

(f)
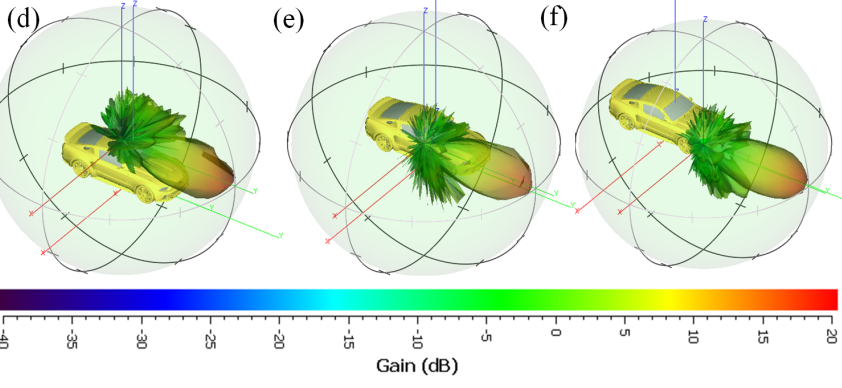

Fig. 5. The scattered radiation pattern. (a) $\sim$ (f) is for the pyramidal horn (vertically aligned corresponding to its E-plane) be located at positions $(1,3,4,5,7)$ and $(8)$ respectively.

pattern shown in Fig. 1 (d) can be placed at different locations on the vehicle's body. This will provide a flexibility for the industry to manufacture the RADAR system without being worried about performance degradation. RADAR systems in today's cars are mostly located in positions (1) or (8) on the bumper which is usually the first part of the automobile that will damaged at car accidents. Apart from that, bumpers are more probable to be covered by mud and slush along the driving that might have a negative impact on the antenna's EM- 
characteristics. This study shows that by considering specific features for the antenna radiation pattern of the autonomous vehicle's RADAR systems, it is feasible to mount the sensors on locations $(4) \sim(6)$ of Fig. 2 where the system will have more chance to be intact at accidents or to remain cleaned most of the times. On the other hand, antenna placing on the car's roof may result the performance being effected by heavy rain or snow. As a result, to increase the safety of passengers, a combination of redundant RADAR system is recommended to guarantee the performance in all conditions.

\section{CONCLUSION}

It is shown how the initial radiation pattern is deformed when the antenna of autonomous car's RADAR sensor is placed on the vehicle body. Two radiation patterns with different gain, HPBW, SLL, and F/B ratio are considered. These two are placed at eight different locations on the body of a realistic Ford Mustang GT model. It is concluded that an antenna with about $19 \mathrm{dBi}$ gain and $-13 \mathrm{~dB}$ SLL (even with relatively improper $\mathrm{HPBW}$ and $\mathrm{F} / \mathrm{B}$ ) can be placed on the bumper, hood, roof, or side-mirror of the car without being concern about the performance degradation of the RADAR system.

\section{REFERENCES}

[1] R. Hussain, S. Zeadally, "Autonomous Cars: Research Results, Issues, and Future Challenges", IEEE Communications Surveys and Tutorials, vol. 21, no. 2, pp. 1275-1313, 2019.

[2] K. Jo, J. Kim, D. Kim, C. Jang, M. Sunwoo, "Development of Autonomous Car-Part II: A Case Study on the Implementation of an Autonomous Driving System Based on Distributed Architecture", IEEE Transaction on Industrial Electronics, vol. 62, no. 8, pp. 5119-5132, 2015.

[3] B. Brown, "The Social Life of Autonomous Cars", Computer, vol. 50, no. 2, pp. 92-96, 2017.

[4] B. S. Lim, S. L. Keoh, V. L. L. Thing, "Autonomous vehicle ultrasonic sensor vulnerability and impact assessment", 2018 IEEE 4th World Forum on Internet of Things (WF-IoT), pp. 231-236, 2018.

[5] A. Stateczny, W. Kazimierski, D. Gronska-Sledz, W. Motyl, "The Empirical Application of Automotive 3D Radar Sensor for Target Detection for an Autonomous Surface Vehicle's Navigation", MDPI remote sensing, vol. 11, pp. 1-18, 2019.

[6] W. Menzel, Antennas in Automobile Radar, Handbook of antenna technologies, Springer, pp. 2475-2500, 2016.

[7] A. Araghi, M. Khalily, P. Xiao, A. Kosari, H. Zarrabi, R. Tafazolli, "Millimeter-Wave MIMO Balanced Antipodal Vivaldi Antenna Design for Autonomous Cars", 2018 International Symposium on Networks, Computers and Communications (ISNCC), 2018.

[8] T. Djerafi, A. Doghri, Ke. Wu, Substrate Integrated Waveguide Antennas, Handbook of antenna technologies, Springer, pp. 1585-1655, 2016.

[9] Available in https://free3d.com/3d-model/ford-mustang-gt-500193364.html.

[10] C. Gopinath, "Automotive Glasses", Session 7, Ramaiah School of Advanced Studies. 